\title{
Application of non-contact magnetic corresponding on the detection for natural gas pipeline
}

\author{
Weiguo Zeng ${ }^{1 *}$, Xiaofeng Dang ${ }^{2}$, Shuhua $\mathrm{Li}^{3}$, Hongmei Wang ${ }^{2}$, Hao Wang ${ }^{2}$ and Bo Wang ${ }^{2}$ \\ ${ }^{1}$ China Special Equipment Inspection and Research Institute, Beijing, 100000, China \\ ${ }^{2}$ The First Gas Production Plant of Changqing Oilfield Branch, Xi'an, Shaanxi, 71000, China \\ ${ }^{3}$ Gas field development department,PetroChina Changqing Oilfield Company, Xi' an, Shaanxi, 71000, China
}

\begin{abstract}
This study examined natural gas pipeline by the non-contact magnetic corresponding. To confirm the reliability for the examination of natural gas pipelines by non-contact magnetic corresponding, three abnormal nodes were detected by the appearance inspection and ultrasonic testing. The results suggested that the grade of abnormal nodes by non-contact magnetic corresponding were not absolutely agree with that of the corrosion by ultrasonic testing. However, there was an obvious relevance between the comprehensive index of $\mathrm{F}$ by non-contact magnetic corresponding and the maximum degree of corrosion by ultrasonic testing. To sum up, the magnetic corresponding was an effective non-contact detection technology for the natural gas pipeline, while it was necessary to rationally grade the abnormal node according to the comprehensive index of $\mathrm{F}$.
\end{abstract}

\section{Introduction}

Detecting the natural gas pipeline was essential for the safe production of natural gas industry[1]. The conventional detecting technologies included the low frequency long distance ultrasonic testing, high frequency guided wave detection, the C-SCAN, ultrasonic wall thickness measurement, magnetic flux leakage testing and the remote field eddy current technology [2]. Although the above technologies could accurately detect the state of natural gas pipelines, they were contacting technologies [3,4]. Therefore, it was necessary to develop a non-contact detection technology, which could meet the acquirement of simple operation and accurate detection.

Recently, the non-contact magnetic corresponding of ferromagnet for the steel in the geomagnetic field had been used for the detection of materials. There were few reports about the application of non-contact magnetic corresponding for petroleum pipeline [5]. While there was no study on the detection by non-contact magnetic corresponding. Herein, this study investigated the application of non-contact magnetic corresponding on the detection of natural gas pipeline. And the reliability by non-contact magnetic corresponding was verified by appearance inspection and ultrasonic testing.

\section{Basic information}

The natural gas pipeline was gas gathering flow line with a length of 6.35 kilometer and an operation pressure of $5.7 \mathrm{Mpa}$, being made of $20 \#$ steel. The diameter and thickness of gas gathering flow line were $114 \mathrm{~mm}$ and 12 mm. Additionally, the it was laid out along the hills (Figure 1).

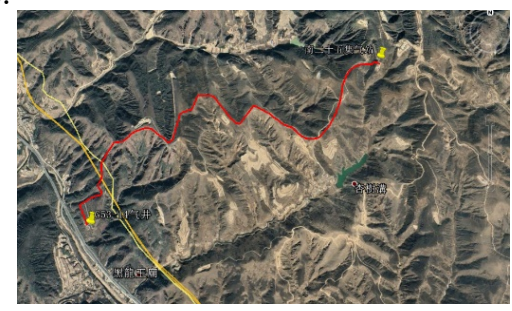

Figure 1. Layout of the gas gathering flow line

\section{Detection by the non-contact magnetic corresponding}

\subsection{Detection index by non-contact magnetic corresponding}

Generally, the degree of defect for a pipeline could be evaluated by the comprehensive index of $\mathrm{F}$ by the non-contact magnetic corresponding, being expressed as in equation (1).

${ }^{*}$ Corresponding author's e-mail: godblesszwg@126.com 


$$
F=A \exp \left(1-Q_{\emptyset} Q_{\mathrm{aH}}^{-1}\right)
$$

Where, $A$ is a corrected coefficient, which implied an influence of geomagnetic field on the pipeline; $Q_{\text {ан }}$ and $Q_{\phi}$ are the densities of magnetic intensity alone with the direction of $\mathrm{Z}$ axle and background of pipeline, respectively.

The degree of defect was graded by the comprehensive index of $\mathrm{F}$ and listed in the Table 1 .

Table 1. Degree of defect and grading for the pipeline by non-contact magnetic corresponding

\begin{tabular}{ccc}
\hline Grade & $\begin{array}{c}\text { Comprehensive index of } \mathrm{F} \text { by the non-contact } \\
\text { magnetic corresponding }\end{array}$ & Degree of defect \\
\hline I & $0 \sim 0.2$ & High \\
II & $0.2 \sim 0.55$ & Medium \\
III & $0.55 \sim 1.0$ & Low \\
\hline
\end{tabular}

\subsection{Results of detection by non-contact magnetic corresponding}

The Figure 2 was the distribution of abnormal nodes for the gas gathering flow line by non-contact magnetic corresponding. It showed that there were 15 abnormal nodes with a grade of II and 63 abnormal nodes with a grade of III, indicating that there was about $20 \%$ of abnormal nodes for the gas gathering flow line was in medium risk.

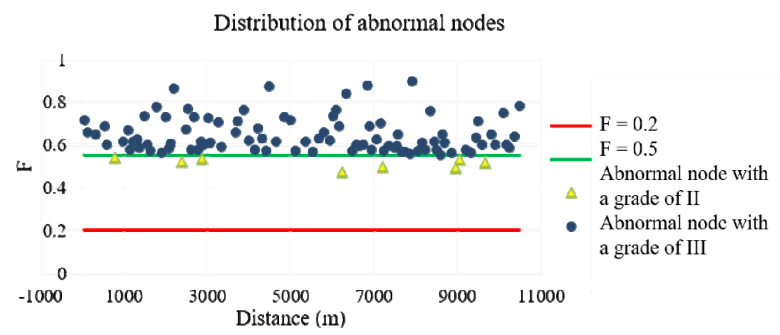

Figure 2. Abnormal nodes for the gas gathering flow line by non-contact magnetic corresponding

\section{Verification}

In order to verify the reliability by the non-contact magnetic corresponding, there were 3 abnormal nodes were examined by the appearance inspection and ultrasonic testing on. It should be noted that the direction of ultrasonic testing was clockwise.

The Figure 3 was the wall thickness distribution by ultrasonic testing and magnetic intensity distribution by non-contact magnetic corresponding for the 3 abnormal nodes. At $1 \#$ abnormal node, the length of pipeline was $0.53 \mathrm{~m}$, the magnetic intensity (DQ) was $981 \mathrm{nT} / \mathrm{m}$, and the comprehensive index of $F$ was 0.575 . The verifying examination showed that the minimum wall thickness was $10.60 \mathrm{~mm}$, corresponding a corrosion degree of $11.67 \%$. The length of abnormal node, DQ and comprehensive index of $\mathrm{F}$ for the $2 \#$ abnormal pipeline were $0.51 \mathrm{~m}, 333 \mathrm{nT} / \mathrm{m}$ and 0.662 , respectively. Similarly, there was a maximum corrosion degree of $6.17 \%$. As for the 3\# abnormal node, the length of abnormal pipeline, DQ and comprehensive index of $F$ were $0.77 \mathrm{~m}, 2365$
$\mathrm{nT} / \mathrm{m}$ and 0.482 , respectively. Although there was no obvious corrosion, the minimum wall thickness was only $9.92 \mathrm{~mm}$, corresponding a reduction degree of $17.33 \%$.
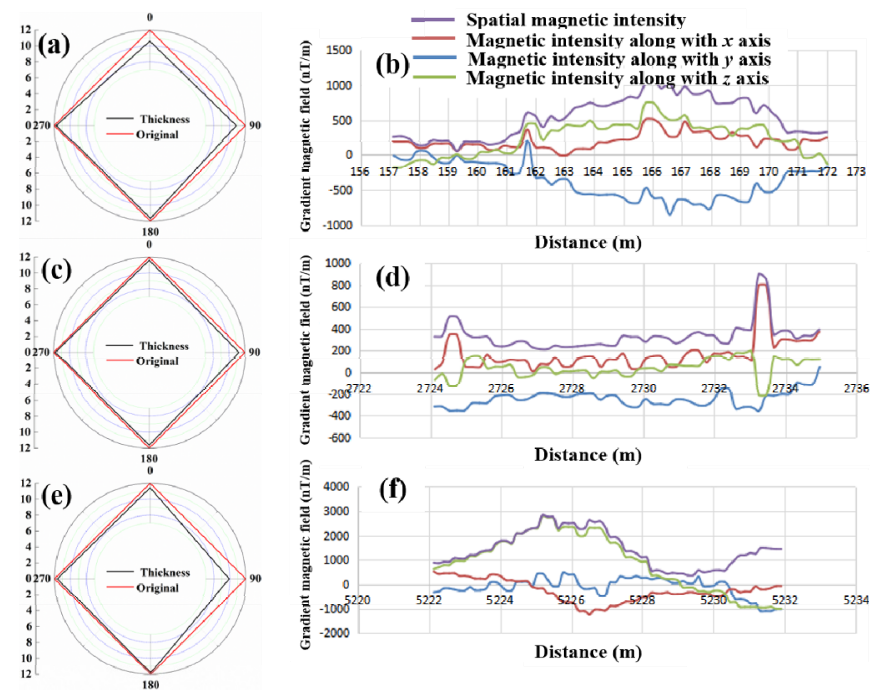

Figure 3. Wall thickness detection by ultrasonic testing and magnetic intensity distribution by non-contact magnetic corresponding for the 3 abnormal nodes ( $a, b$ and $\mathrm{c}$ were the results of $1 \#, 2 \#$ and $3 \#$ abnormal node, respectively.)

\section{Analysis}

The appearance inspection and ultrasonic testing suggested that the wall thickness reduction was a common phenomenon. According to the standard of $\mathrm{SY} / \mathrm{T}$ 0087.2, the corrosion degree of $1 \#$ and 3\# abnormal nodes were middle, while the corrosion degree of $2 \#$ abnormal node was light. However, the detection by non-contact magnetic corresponding indicated that the degree of defection for $1 \#$ and 2\# abnormal nodes were middle, but it was light for $3 \#$ abnormal node. Therefore, the grading by non-contact magnetic corresponding for the gas gathering flow line was not agree well with that of conventional technology.

However, it was also found that the comprehensive 
index of $\mathrm{F}$ was negatively relative with the maximum reduction degree for the gas gathering flow line significantly (Figure 4).

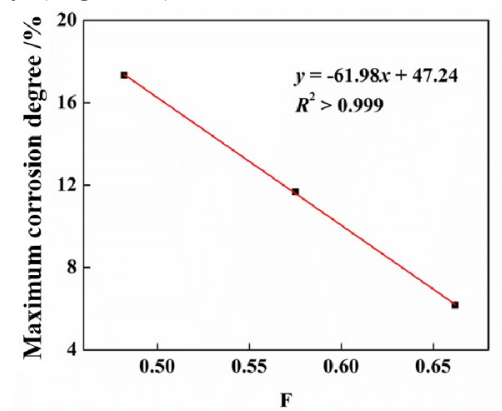

Figure 4. Linear fitting between the comprehensive index of $\mathrm{F}$ by non-contact magnetic corresponding and the maximum corrosion degree by ultrasonic testing for the G-A gas gathering flow line

\section{Conclusion}

In summary, this study applicated the non-contact magnetic corresponding on the detection of natural gas pipeline (G-A gas gathering flow line). It was found that there were few abnormal nodes with a grade of II and a lot of nodes with a grade of III, while without the abnormal node with a grade of I. Although the grading by the non-contact magnetic corresponding for the G-A gas gathering flow line was not agree well with that of verified examination by ultrasonic testing, there was a significant correlation between the comprehensive index of $\mathrm{F}$ and the maximum corrosion degree for the G-A gas gathering flow line. Therefore, the non-contact magnetic corresponding could be used for the detection of natural gas pipeline with a condition of corrected grading according to the special natural gas pipeline.

\section{Acknowledgments}

The work was financially supported by the Research on regular inspection strategy of natural gas purification plant pipeline ( K-19-D02 ) and Study on external corrosion rate model of buried oil and gas pipeline(2016 nei 17).

\section{Reference}

1. Yang, Q.X., Wen, X.C. (2019) Analysis on formation of corrosion of long-distance natural gas pipeline and corrosion protection measures. Yunnan Chemical Technology, 46: 124-125.

2. Zhao, M.L., Zhang, J.J., Zhang, S.Q., Yang, B. (2014) Corrosion of gathering pipelines of single well in Yakela gas field and testing. Corrosion \& Protection in Petrochemical Industry, 31: 48-52.

3. Tao, W.J., Yang, Z.W., Wu, X.Q., Hu, C.Y., Yan, M.C., Yu, C.K., Xu, J., Sun, C. (2017) Comprehensive approach of inspection and assessment of external corrosion of aged pipelines in oil/gas field.
CorrosionScience and Protection Technology, 29: 188-194.

4. Tang, S., Wang, C.Y. (2017) Corrosion detection snd evaluation for oil and gas pipelines. Petrochemical Industry Technology, 24: 107-108+98.

5. Xu, S.N., Jiang, H., Li, J.W., Zhang, J. (2017) Application for buried pipeline of non-excavation high-precision magnetic stress inspection and evaluation technology. Total Corrosion Contral, 31: 26-28. 